\title{
Síntese de conversores MMC para aplicação em sistemas de geração fotovoltaicos e sistemas de transmissão em corrente contínua ${ }^{1}$
}

\author{
Synthesis of MMC converters for photovoltaic generating systems and direct \\ current transmission systems applications
}

\author{
Andrei de Oliveira Almeida ${ }^{2}$ \\ Bárbara da Silva Medeiros ${ }^{3}$ \\ João Pedro Peters Barbosa ${ }^{4}$ \\ Pedro Gomes Barbosa ${ }^{5}$
}

DOI: https://doi.org/10.34019/2179-3700.2019.v19.29893

\begin{abstract}
Resumo
Este trabalho apresenta um estudo sobre a utilização do conversor multinível modular no processamento da energia elétrica gerada por fontes renováveis (e.g. solar, eólica, entre outras) ou na transmissão de energia elétrica em corrente contínua. Conversores multiníveis modulares foram originalmente propostos para aplicações em altas tensões devido à possibilidade de expansão do número de níveis da tensão terminal de saída. Apesar do foco da proposta inicial, esses conversores se mostraram interessantes para serem utilizados em sistemas com tensões mais baixas, eliminando a necessidade de transformadores complexos ou filtros harmônicos de ordem elevada. Como a operação dos conversores multiníveis modulares dependente da equalização das tensões dos capacitores dos seus diversos submódulos, este artigo apresenta um estudo comparativo de diferentes métodos de ordenação usados para selecionar os submódulos de acordo com o valor das tensões dos seus capacitores. Resultados de simulação obtidos com o software de transitórios eletromagnéticos são apresentados para demonstrar o desempenho das estratégias de ordenação e controle investigadas. O esforço computacional dos algoritmos de ordenação investigados são também testados experimentalmente utilizando um processador digital de sinais.
\end{abstract}

Palavras-chave: Conversor Multinível Modular. Equalização das Tensões. Métodos de Ordenação. Algoritmos de Ordenação. Simulação Digital.

\begin{abstract}
This paper presents a study on the use of the modular multilevel converter in the processing of the electric energy generated by renewable sources (e.g. solar, wind, among others) or in the electric energy transmission in direct current. Modular multilevel converters were originally proposed for applications at high voltages due to the possibility of expanding the number of output terminal voltage levels. Despite the initial proposal focus, these converters have proved to be of interest for

\footnotetext{
${ }^{1}$ Trabalho premiado no Seminário de Iniciação Científica da UFJF em 2016.

2 Bolsista do programa PROBIC/FAPEMIG - E-mail: andrei.almeida@engenharia.ufjf.br

${ }^{3}$ Bolsista do programa BIC/UFJF - E-mail: barbara.medeiros@engenharia.ufjf.br

${ }^{4}$ Bolsista do programa BIC/UFJF - E-mail: joao.peters@engenharia.ufjf.br

${ }^{5}$ Professor orientador; Departamento de Energia Elétrica - UFJF - E-mail: pedro.gomes@ufjf.edu.br
} 
use in systems with lower voltages, eliminating the need for complex transformers or high-order harmonic filters. As the operation of the modular multilevel converters depends on the equalization of the capacitor voltages of its several submodules, this paper presents a comparative study of different sorting methods used to select the submodules according to the voltage value of their capacitors. Simulation results obtained with the software of electromagnetic transients are presented to demonstrate the performance of the sorting and control strategies investigated. The computational effort of the investigated sorting algorithms will also be experimentally tested using a digital signal processor.

Keywords: Modular Multilevel Converter. Voltage Equalization. Sort Methods. Sort Algorithms. Digital Simulation.

\section{INTRODUÇÃO}

A demanda por novas fontes de energia tem levado engenheiros e cientistas a buscarem formas mais eficientes de geração, transmissão e distribuição de eletricidade. Dentro deste contexto tem-se observado uma tendência da utilização de sistemas de geração distribuída (SGD) baseados em fontes renováveis (e.g. energia solar, eólica, entre outras) (CARRASCO et al., 2006). Contudo, devido à natureza unidirecional ou intermitente de muitas dessas fontes alternativas, a conexão desses SGD em paralelo com a rede elétrica de distribuição só é possível com uso de conversores eletrônicos de potência (MOHAN, 2002).

A Figura 1 mostra a topologia básica de um conversor fonte de tensão trifásico (VSC - do inglês, Voltage Source Converter) em ponte completa, que tem conectado em seus terminais CC um painel fotovoltaico. Este conversor, cujas tensões trifásicas de saída possuem dois níveis, é composto por seis interruptores do tipo IGBT (do inglês, Insulated Gate Bipolar Transistor) com seis diodos conectados em antiparalelo. Neste exemplo o conversor estático processa a energia CC gerada pelo painel fotovoltaico transformando-a em CA.

Figura 1 - Topologia básica de um conversor CC-CA trifásico com tensão de saída com dois níveis

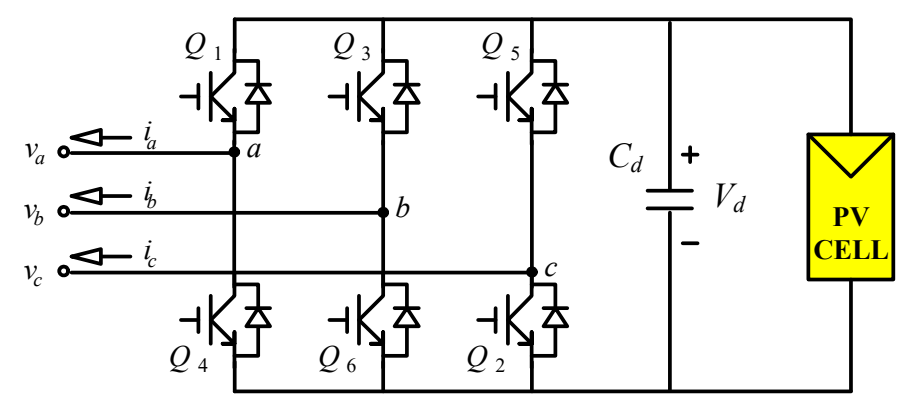

Fonte: o autor 
Filtros passivos de primeira, segunda e terceira ordens ( $L, L C$ e $L C L)$ são normalmente conectados nos terminais CA dos VSC (TEODORESCU; LISERRE; RODRÍGUEZ, 2011) para evitar que os harmônicos gerados pelas comutações dos IGBT sejam injetados na rede elétrica (IEEE 929, 2000) e (IEC 61727, 2004). Além dos filtros harmônicos são também conectados transformadores aos terminais dos VSC para ajustar o nível das tensões sintetizadas e isolar galvanicamente seus terminais de saída.

Como alternativa ao uso dos conversores com tensão de saída com dois níveis, com filtros e transformadores elevadores conectados aos seus terminais, pode-se usar conversores cujas tensões terminais têm múltiplos níveis. Na Figura 2(a) é mostrada a topologia do conversor multinível modular (MMC - do inglês, Modular Multilevel Converter). Conforme pode-se observar nessa figura, cada braço do MMC é formado pela associação série de $N$ submódulos (SM) e um indutor $L_{0}$, sendo que cada fase do conversor é formada pela associação de dois braços, um superior e um inferior. Diferentes topologias de unidades conversoras podem ser usadas para sintetizar os SM.

Na Figura 2(b) é ilustrada a topologia de um SM baseado no conversor em meia ponte usada neste trabalho. Esta unidade conversora contém dois IGBT e um capacitor CC (SHARIFABADI et al., 2016). Os interruptores S1 e S2 são acionados de maneira complementar, de modo que quando um conduz o outro é colocado no estado de corte e vice-versa. Um SM é dito ativo quando S1 está conduzindo e S2 está cortado. Nesta condição o capacitor $\mathrm{CC}$ pode se carregar ou descarregar, dependendo do sentido da corrente que flui pelo SM, como ilustrado nas Figuras 2(b) e 2(c), respectivamente. 
Figura 2 - (a) Diagrama esquemático do MMC, (b) SM ativo com a carga do capacitor CC e (c) SM ativo com a descarga do capacitor CC.

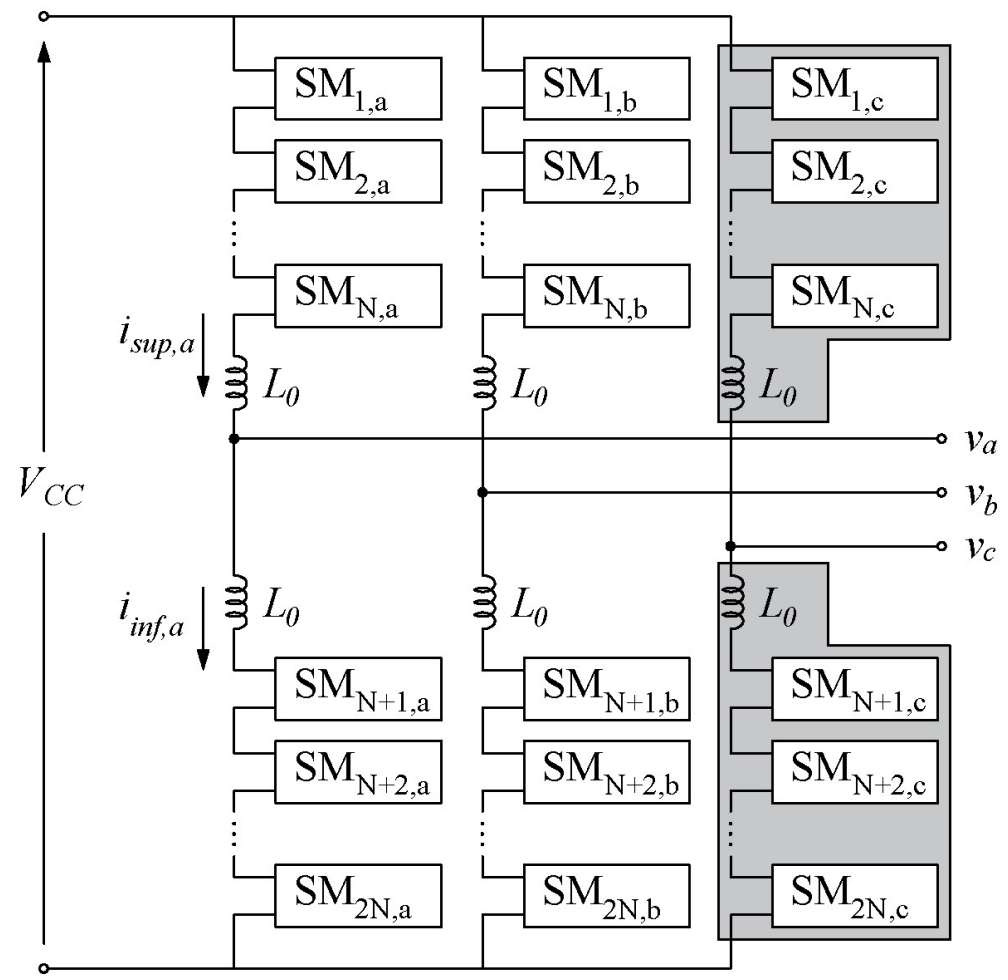

(a)

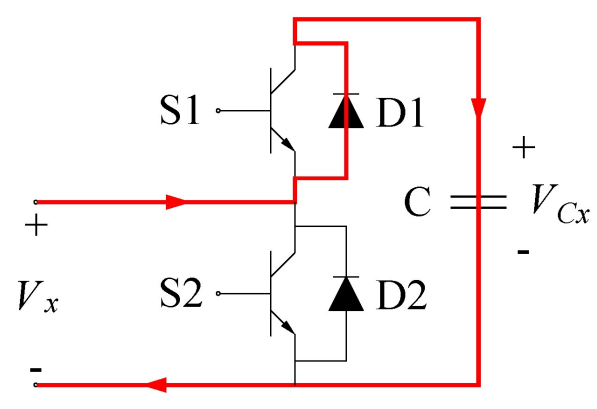

(b)

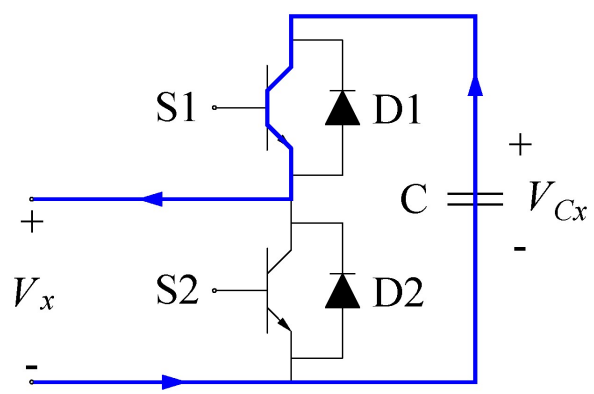

(c)

Fonte: Autor

Historicamente O MMC foi proposto por Lesnicar e Marquardt (2003) para aplicações em sistemas de transmissão em corrente contínua e alta tensão (HVDC - do inglês, High Voltage Direct Current). Contudo, a estrutura modular deste conversor se mostrou também interessante para aplicações em médias tensões (DEBNATH et al., 2015). No caso de um SGD fotovoltaico de média ou elevada capacidade, a conexão de painéis fotovoltaicos à rede elétrica de distribuição normalmente é feita pela associação em paralelo de vários VSC semelhantes ao da Figura 1, com seus filtros harmônicos e transformadores. Por outro lado, todos esses VSC, com tensão de saída com dois níveis, poderiam ser substituídos por um único conversor MMC. A utilização do MMC eliminaria a necessidade do transformador e, como o conteúdo harmônico das tensões sintetizadas pelo MMC é baixo, os filtros passivos usados para mitigar os harmônicos de comutação do conversor seriam menores ou poderiam ser eliminados.

Paralelamente aos desafios anteriores, novas tecnologias vêm sendo estudadas para aumentar a eficiência dos sistemas de transmissão e distribuição da energia elétrica. Um exemplo são os sistemas de transmissão HVDC baseados em VSC (ARRILLAGA; 
LIU; WATSON, 2007). Os VSC-HVDC apresenta uma maior controlabilidade e menores perdas quando comparados aos sistemas de transmissão HVDC convencionais baseados em conversores fonte de corrente (CSC - do inglês, Current Source Converters). Esses conversores também facilitam a construção de sistemas HVDC multiterminais.

Sendo assim, o principal objetivo deste trabalho é investigar o uso do conversor multinível modular para processar e condicionar a energia elétrica gerada por sistemas de geração alternativos de média e grande capacidades, especialmente os SGD baseados em painéis fotovoltaicos. Durante a operação do MMC, erros e atrasos de chaveamento provocam o desvio das tensões CC dos diferentes SM da estrutura. Caso nenhuma técnica de correção seja aplicada, esse comportamento faz com que as tensões nos capacitores do MMC se desviem do ponto de operação nominal, comprometendo a segurança do conversor. Desse modo, para garantir o funcionamento estável deste conversor é necessário regular e equalizar as tensões dos diversos capacitores dos SM. Nesse sentido, os desempenhos de diferentes métodos de ordenação aplicados ao algoritmo de equalização das tensões nos capacitores do MMC serão modelados, simulados e testados em um programa digital de transitórios eletromagnéticos e com auxílio de um processador digital de sinais (DSP - do inglês, Digital Signal Processor).

\section{ALGORITMO DE EQUALIZAÇÃO DAS TENSÕES DOS CAPACITORES}

Conforme mencionado anteriormente, erros e atrasos de chaveamentos dos IGBT e não linearidades provocam o desvio das tensões $C C$ dos capacitores dos vários SM do MMC durante a operação do conversor. Essas tensões, por sua vez, não são equalizadas naturalmente e devem ser reguladas para evitar algum dano ou mal funcionamento (ALMEIDA et al, 2016).

Duas estratégias básicas são propostas na literatura para regular e equalizar as tensões CC dos diferentes SM: (i) na primeira, a tensão de cada capacitor é controlada individualmente, alterando o padrão de chaveamento dos interruptores de cada SM (GLINKA, 2004); (ii) na segunda, utiliza-se um algoritmo de ordenação e seleção das tensões de cada submódulo para escolher quais deverão ficar ativos em determinado instante de tempo (SAEEDIFARD; IRAVANI, 2010). A principal vantagem da segunda estratégia é o fato desta não necessitar de circuitos adicionais, já que é implementada junto com o sistema de controle e disparo dos interruptores do MMC.

Pelo exposto, na implementação da segunda estratégia de equalização das 
tensões dos capacitores do MMC, pode-se utilizar diferentes métodos de ordenação para ranquear os diferentes SM em função das tensões $\mathrm{CC}$ dos seus capacitores. Entretanto, não há um estudo na literatura indicando qual método de ordenação é o mais indicado ou eficiente para o MMC. Nesse sentido, nesta seção serão comparados os desempenhos de diferentes métodos de ordenação aplicados ao algoritmo de equalização das tensões nos capacitores do MMC.

O algoritmo de equalização começa pela determinação do número de SM que deve estar ativo em cada braço do MMC em cada instante de tempo. Para isso, utiliza-se uma estratégia de modulação onde múltiplas portadoras triangulares, deslocadas em nível, são comparadas com o sinal de referência senoidal que se deseja sintetizar nos terminais de saída do conversor (YAZDANI; IRAVANI, 2010). Pode-se usar também uma estratégia em que as múltiplas portadoras triangulares são deslocadas em fase (RAJAN; SEYEZHAI, 2013).

No caso do MMC, deve-se utilizar um número de portadoras triangulares igual ao número de SM por braço $(N)$. A tensão terminal de saída poderá ter $(N+1)$ ou $(2 N+1)$ níveis diferentes dependendo de como será feita a comparação dos sinais. Neste trabalho utilizou-se a estratégia $(N+1)$, de maneira que a soma dos SM ativos nos braços superior e inferior de cada fase é sempre constante e igual ao número de submódulos por braço, ou seja, N. Na Figura 3(a) é mostrada a comparação entre o sinal de referência senoidal e quatro portadoras triangulares deslocadas em nível. Já na Figura 3(b) é mostrado o número de SM ativos no braço superior resultante dessa comparação. Determinado o número de SM que devem estar ativos nos braços de cada fase, pode-se então escolher quais serão ativados ou não.

Na Figura 4 é mostrado um fluxograma com o algoritmo para a regulação das tensões CC dos capacitores do MMC. Conforme explicado anteriormente é usado um algoritmo para ordenar um vetor com as tensões dos diferentes capacitores do braço positivo e negativo de cada fase do conversor. Uma estratégia de modulação semelhante à mostrada na Figura 3(a) é usada para determinar quantos SM deverão estar ativos nos braços superior $\left(n_{\text {sup }}\right)$ e inferior $\left(n_{\text {inf }}\right)$ em cada instante de tempo, respectivamente. De posse do número de SM ativos e do vetor ordenado das tensões CC dos diversos SM passa-se à etapa de seleção de quais SM deverão ser inseridos no circuito. Para realizar essa tarefa deve-se considerar o sentido das correntes $i_{\text {sup }}$ e $i_{\text {inf }}$ que fluem pelo braços superior e inferior de cada fase do conversor, respectivamente. 
Figura 3 - Ilustração da estratégia de modulação usada para determinar o número de SM ativos no braço superior de cada fase do MMC: (a) sinal de referência senoidal e portadoras triangulares e (b) número de SM ativos

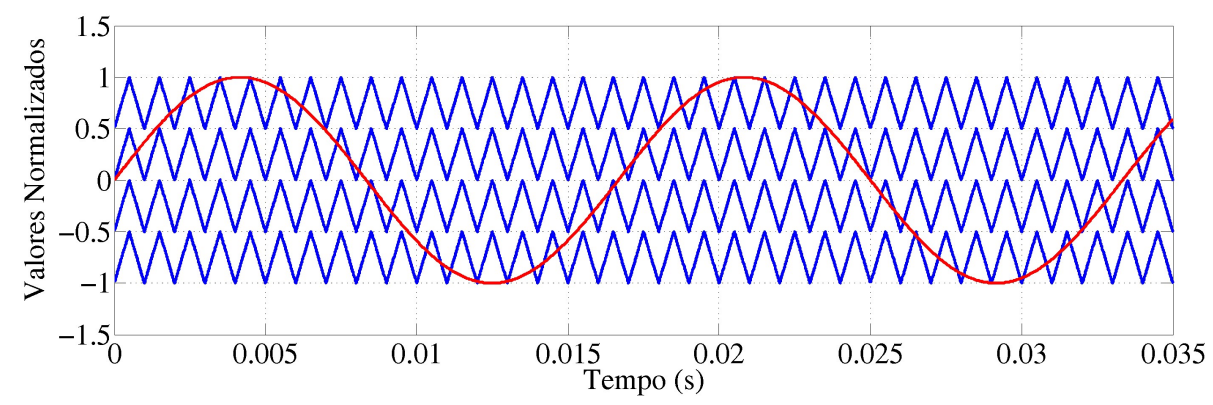

(a)

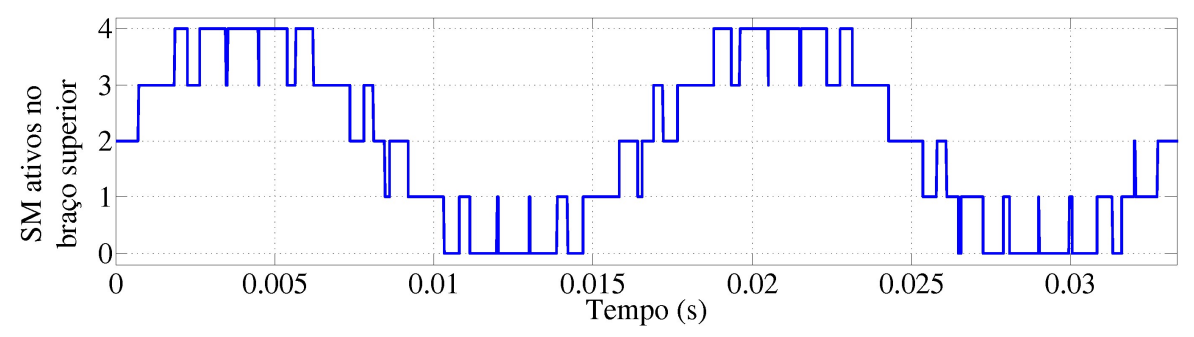

(b)

Fonte: o autor

Figura 4 - Fluxograma do algoritmo de regulação e equalização das tensões nos capacitores do MMC

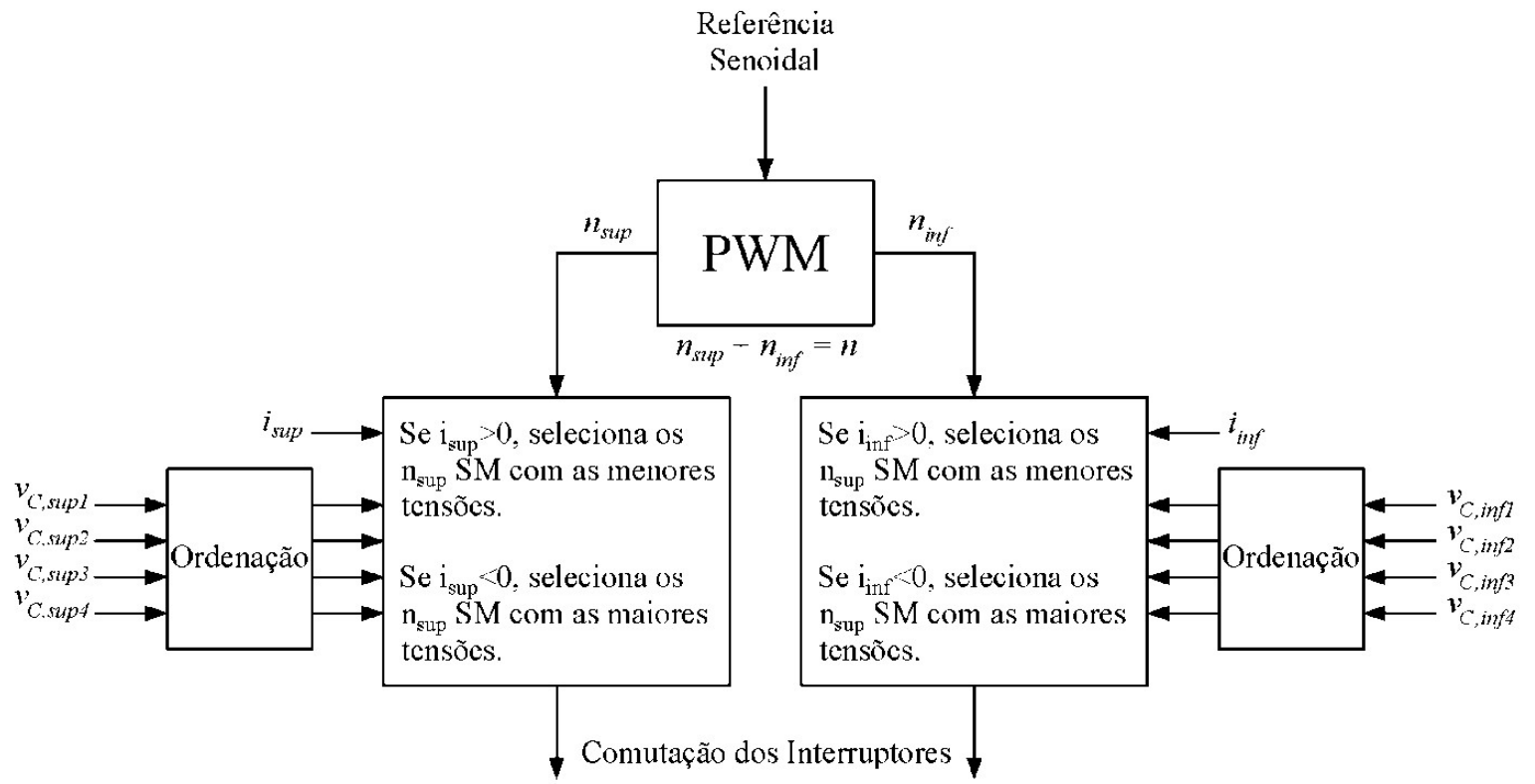

Fonte: Almeida et al (2016). 
Se a corrente no braço é positiva, deverão ser selecionados os SM com capacitores menos carregados, para que estes carreguem e elevem suas tensões. Esta condição é a mesma mostrada na Figura 2(b). Por outro lado, quando a corrente é negativa, devem ser selecionados os SM mais carregados para que os mesmos se descarreguem e diminuam suas tensões. A Figura 2(c) mostra o sentido da corrente pelo capacitor de um SM nesta condição.

Conforme mencionado anteriormente, pode-se utilizar diferentes métodos de ordenação para ranquear os diversos SM do conversor em função das tensões CC de seus capacitores. Saeedifard e Iravani (2010) utilizaram o método de ordenação da bolha. Apesar de simples, esse método não é o mais eficiente, principalmente quando o número de SM do MMC é elevado. A seguir serão apresentados de maneira sucinta os princípios de funcionamentos dos principais métodos de ordenação analisados nesta pesquisa.

\subsection{Método da Bolha}

Para ordenar um vetor de $n$ posições, o método da bolha percorre o vetor $n-1$ vezes, comparando elementos adjacentes e realizando a troca dos elementos caso seja necessário. Na Figura 5 é ilustrada a ordenação de um vetor de quatro posições pelo método da bolha. Note que, após a primeira iteração, o maior elemento vai para a última posição e assim por diante, até que o menor elemento ocupe a primeira posição e o vetor esteja ordenado.

Figura 5 - Exemplo de ordenação pelo método da bolha

$$
\begin{aligned}
& \begin{array}{|l|l|l|l|}
\hline 4 & 9 & 7 & 2 \\
\hline
\end{array} \\
& \begin{array}{|l|l|l|l|}
\hline 4 & 9 & 7 & (2) \\
\hline
\end{array} \\
& \begin{array}{|l|l|l|l|}
\hline 4 & 7 & 9 & 2 \\
\hline
\end{array} \\
& \begin{array}{|l|l|l|l|}
\hline 4 & 7 & 2 & 9 \\
\hline
\end{array} \\
& \begin{array}{|l|l|l|l|}
\hline 4 & 2 & 7 & 9 \\
\hline
\end{array} \\
& \begin{array}{l|l|l|l|}
\hline 2 & 4 & 7 & 9
\end{array} \quad \text { Vetor Final }
\end{aligned}
$$

Fonte: Almeida et al (2016). 


\subsection{Método da Inserção}

Nesse método, a primeira iteração analisa o segundo elemento do vetor e, se este for menor que o primeiro, eles trocam de posição. Na segunda iteração, o terceiro elemento é comparado com o segundo (se for menor trocam de posição, se não a iteração acaba). Se a troca ocorrer, uma nova comparação é feita com o primeiro elemento, e o mesmo procedimento ocorre. Esse processo ocorre até o último elemento do vetor, como ilustrado na Figura 6.

Figura 6 - Exemplo de ordenação pelo método da inserção

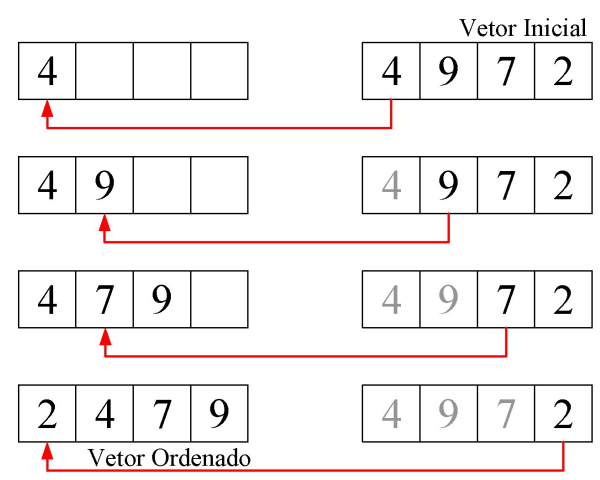

Fonte: Almeida et al (2016).

\subsection{Método da Seleção}

Na primeira iteração, o método da seleção percorre todo o vetor em busca do menor elemento, que é trocado com o primeiro elemento caso não seja este o menor. $\mathrm{Na}$ segunda iteração, o vetor é percorrido do segundo até o último elemento, de maneira que o menor elemento desta iteração vá para a segunda posição. O mesmo ocorre nas demais iterações, como ilustrado na Figura 7. 
Figura 7 - Exemplo de ordenação pelo método da seleção

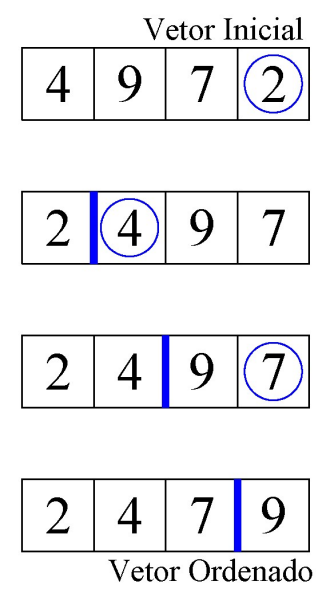

Fonte: Almeida et al (2016).

\subsection{Método Shell}

Esse método é inspirado no método da inserção. Contudo, o método Shell é mais eficiente quando os vetores são muito grandes. Ao contrário da inserção, que compara apenas elementos adjacentes, o método Shell permite comparar elementos separados por uma distância $h$, que deve seguir uma sequência decrescente até $h=1$, quando o algoritmo se torna equivalente ao da inserção. Um exemplo de sequência é dado por:

$$
h(n)=\left\{\begin{array}{c}
3 h(n-1)+1, \text { paran }=2,3,4, \ldots \\
1, \text { paran }=1 .
\end{array}\right.
$$

No método da inserção, quando o menor elemento está na última posição, são necessárias $n-1$ trocas para que ele chegue à primeira posição. Já o método Shell evita esse problema comparando elementos distantes.

\subsection{Método da Fusão}

A ideia desse método é dividir o vetor em dois ou mais subvetores e ordená-los por um método mais simples, depois realizar a fusão desses vetores menores. Para vetores com muitos elementos, essa estratégia pode acelerar o processo de ordenação. $\mathrm{Na}$ Figura 8 é ilustrado um exemplo de ordenação de um vetor de seis posições pelo método da fusão. 
Figura 8 - Exemplo de ordenação pelo método da fusão

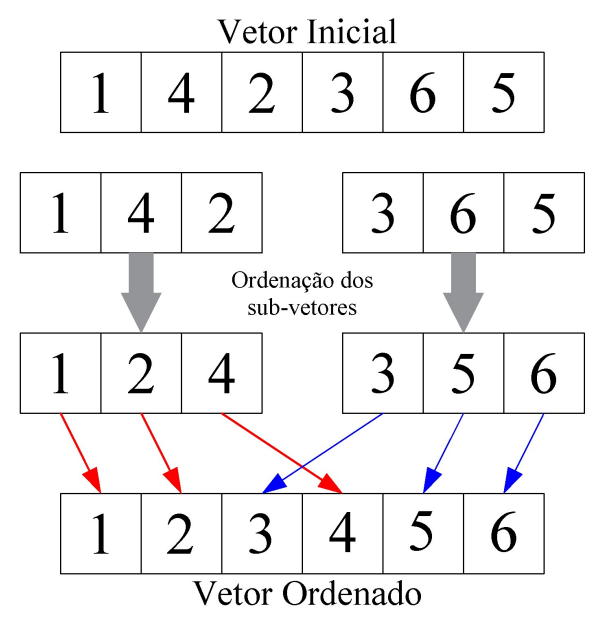

Fonte: Almeida et al (2016).

\subsection{Método Quick}

Este último método é muito semelhante ao método da fusão, porém a divisão do vetor é feita de maneira diferente. No método Quick, é escolhido um elemento "pivô" e vetor é dividido em dois subvetores com elementos menores e maiores que o pivô. Com os subvetores ordenados, não é necessário realizar a fusão, já que todos os elementos do primeiro subvetor são menores que os do segundo. Assim como no método da fusão, é possível dividir os subvetores sucessivamente. Apesar dessas vantagens, o desempenho do método Quick é muito sensível à escolha do pivô, que pode ser feita de várias formas.

A seguir serão mostrados os resultados das simulações com o objetivo de verificar o funcionamento do algoritmo de equalização, assim como os resultados dos métodos de ordenação implementados no DSP.

\section{RESULTADOS DE SIMULAÇÃO E DOS TESTES NO DSP}

Com o objetivo de verificar o desempenho dos diferentes algoritmos de ordenação estudados para regular as tensões dos capacitores do MMC, foi modelado e simulado no programa PSIM um sistema de transmissão HVDC com dois MMC, como ilustrado de forma esquemática na Figura 9. Os dois MMC, um retificador e o outro inversor, foram montados com quatro SM por braço. 
Figura 9 - Diagrama esquemático de um sistema de transmissão MMC-HVDC

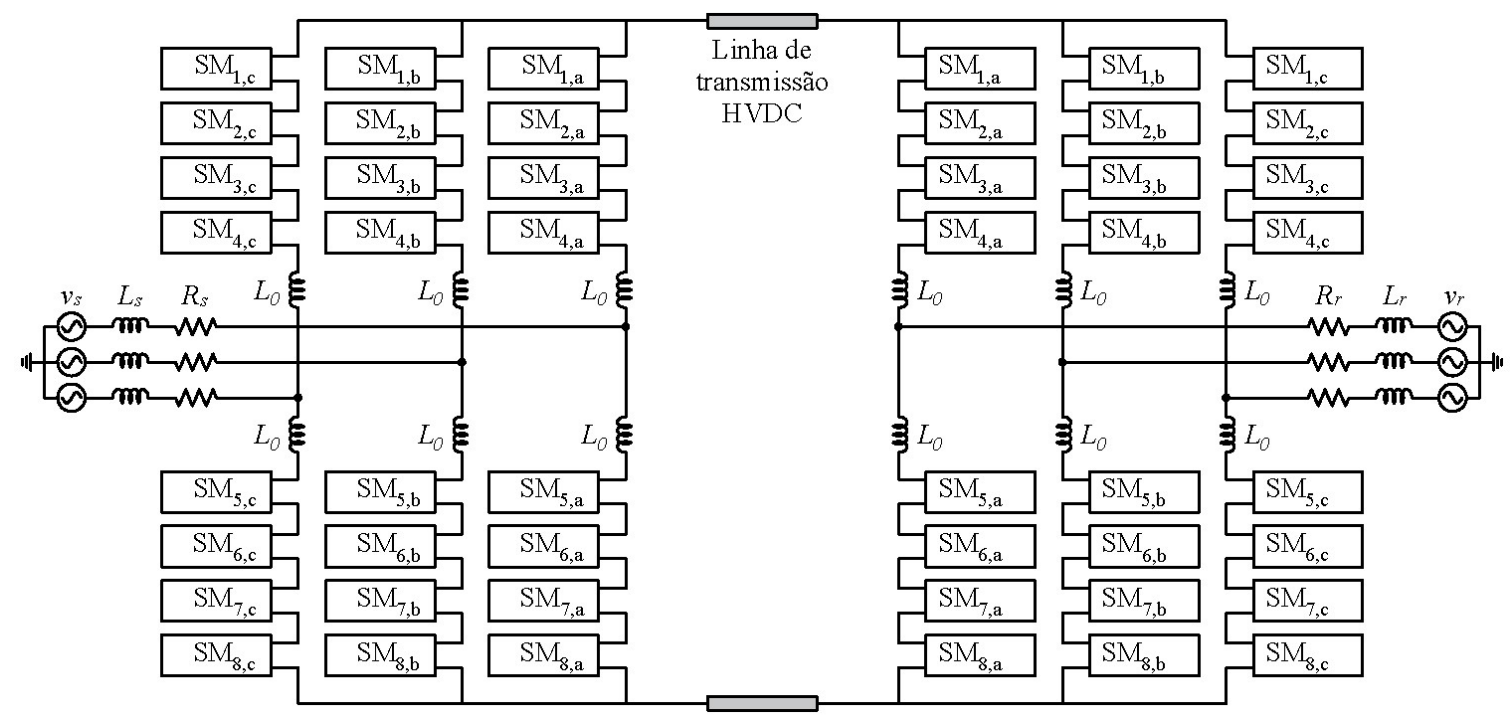

Fonte: o autor.

Os dois conversores da Figura 9 estão interligados por uma linha de transmissão CC. O MMC da esquerda (retificador) é conectado, através de uma indutância $L_{s}$ e uma resistência $R_{S}$, a uma rede trifásica com tensões $v_{s}$. Do outro lado, o MMC da direita (inversor) é conectado a outra rede trifásica que recebe a potência transmitida pela linha CC e a injeta na rede trifásica com tensões $v_{r}$. Além do algoritmo de equalização, foram usados diferentes controladores para regular a potência fornecida pelo retificador e para regular a tensão CC na linha, através do inversor (YAZDANI; IRAVANI, 2010). Na Tabela 1 são listados os parâmetros utilizados nas simulações.

Tabela 1 - Parâmetros do sistema simulado

\begin{tabular}{lr}
\hline \multicolumn{1}{c}{ Parâmetro } & \multicolumn{1}{c}{ Valor } \\
\hline Potência nominal $\left(P_{s}\right)$ & $15 \mathrm{kVA}$ \\
Tensão nominal de linha $\left(V_{s} \mathrm{e} V_{r}\right)$ & $13,8 \mathrm{kV}$ \\
Frequência das redes $\left(f_{s}\right.$ e $\left.f_{r}\right)$ & $60 \mathrm{~Hz}$ \\
Frequência das portadoras triangulares $\left(f_{t r i}\right)$ & $1200 \mathrm{~Hz}$ \\
Indutância dos braços $\left(L_{0}\right)$ & $0,3 \mathrm{mH}$ \\
Indutância dos filtros $\left(L_{s}\right.$ e $\left.L_{r}\right)$ & $1,7 \mathrm{mH}$ \\
Resistência dos filtros $\left(R_{s}\right.$ e $\left.R_{r}\right)$ & $0,4 \Omega$ \\
Capacitância dos SM $(C)$ & $1000 \mu \mathrm{F}$ \\
Indutância da linha de transmissão & $10 \mathrm{mH}$ \\
Resistência da linha de transmissão & $0,5 \Omega$ \\
\hline
\end{tabular}

Fonte: o autor. 
$\mathrm{Na}$ Figura 10, em (a), (b) e (c) são mostradas as formas de onda das potências ativa e reativa instantâneas e das tensões nos capacitores do MMC retificador sem e com o algoritmo de equalização, respectivamente. Em $t=0,02 \mathrm{~s}$ os conversores iniciam sua operação, porém sem injetar ou consumir potência da rede. Em $t=0,1 \mathrm{~s}$, o sinal de referência da potência ativa instantânea do retificador é variado em degrau de 0 para 1 pu (valor nominal). A partir deste instante o retificador passa a consumir potência ativa da rede $\mathrm{CA}$ e injetar na linha de transmissão $\mathrm{CC}$. $\mathrm{Em} t=0,2 \mathrm{~s}$, a potência reativa instantânea é variada em degrau, porém agora de 0 para $-0,6$ pu. A observação da Figura 10 (b) permite concluir que quando o retificador começa a transmitir potência ativa pela linha $\mathrm{CC}$, as tensões dos capacitores dos SM divergem de seu valor nominal ( $0,5 \mathrm{pu})$ quando não é usado o algoritmo de equalização no controle dos conversores. Observa-se que algumas tensões CC ultrapassam 0,5 pu, o que pode comprometer a operação do sistema, já que os SM não são projetados para suportar tais sobretensões. Já na Figura 10 (c) é mostrado o comportamento das tensões CC dos capacitores dos SM quando é usado o algoritmo de regulação e equalização das tensões.

Os resultados mostrados na Figura 10 foram obtidos nas simulações com o método de ordenação da bolha, assim como na proposta inicial. Porém, todos os métodos foram testados em simulações e apresentaram resultados semelhantes, não afetando a operação dos conversores. A seguir serão mostrados os resultados dos testes feitos com os demais métodos de ordenação no DSP. 
Figura 10 - Formas de onda do retificador MMC: (a) potências ativa e reativa instantâneas; (b) tensões nos capacitores sem a estratégia de equalização e (c) tensões nos capacitores com a estratégia de equalização

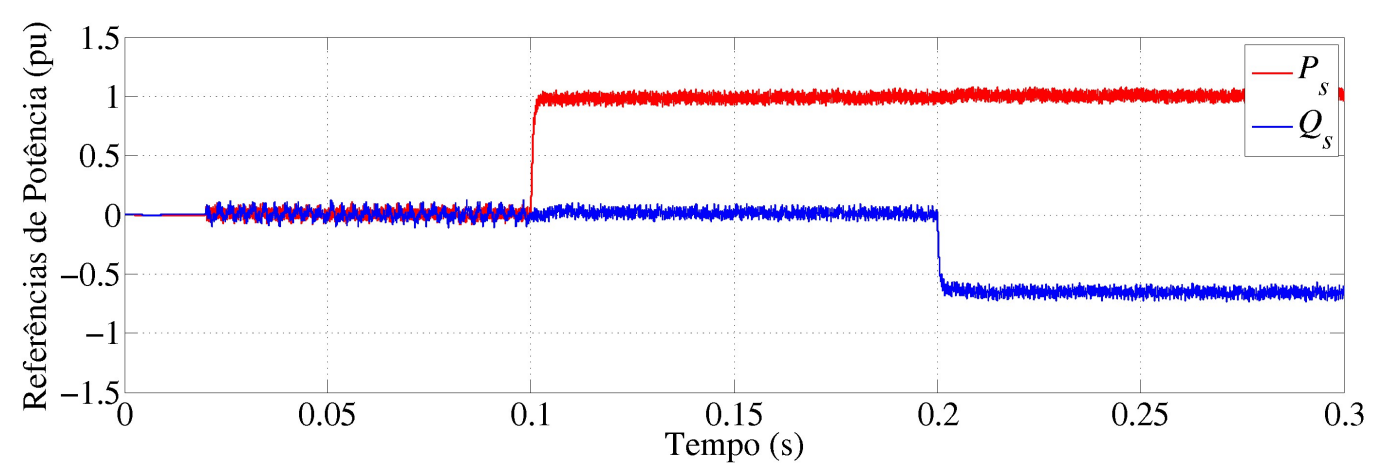

(a)

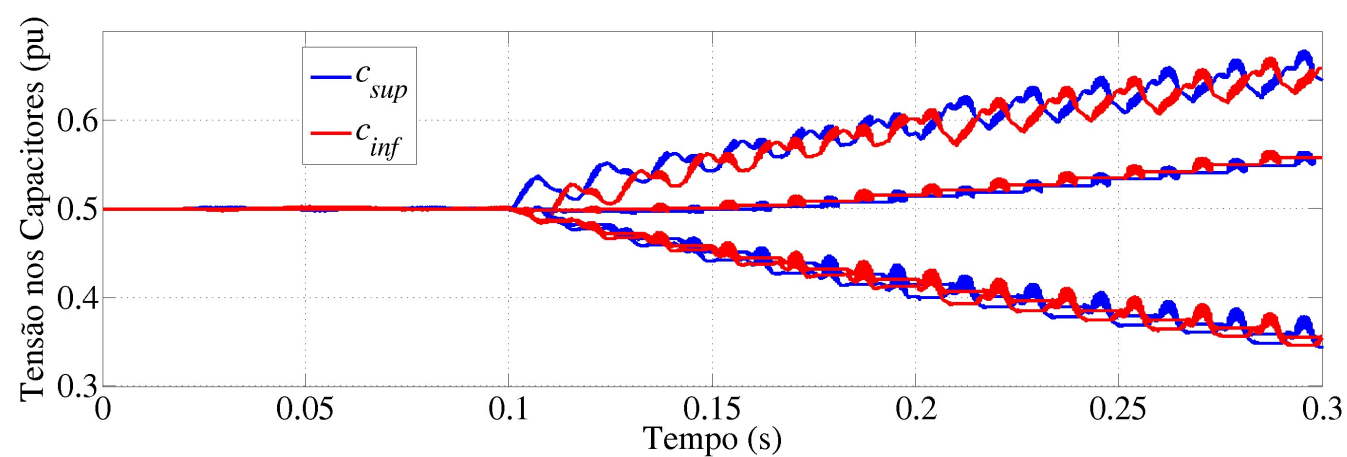

(b)

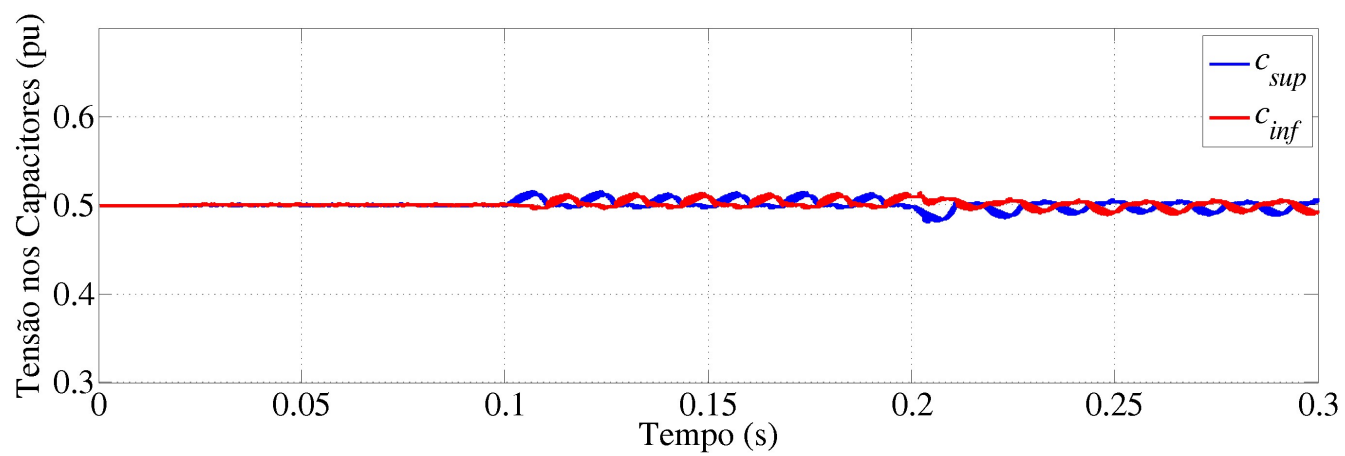

(c)

Fonte: o autor.

Posteriormente, o algoritmo de equalização das tensões dos capacitores $\mathrm{CC}$, mostrado na forma de diagrama de blocos na Figura 4, foi implementado em um DSP TMS320F28335 da Texas Instruments, com os seis métodos de ordenação apresentados na Seção 2. Foram então medidos os tempos de processamento com o objetivo de quantificar o esforço computacional de cada um dos métodos investigados nesse trabalho. Para tal foram testados 
o melhor caso, onde o vetor de tensões já estava ordenado, e o pior caso, onde o vetor de tensões está ordenado inversamente. Além disso, foram testados casos com diferentes números de SM por braço. Começando com quatro SM por braço, como nas simulações, e chegando a dezesseis. Com isso foi possível observar o comportamento do tempo de processamento do algoritmo a medida que o tamanho do vetor de tensões era aumentado.

Na Figura 11, em (a) e (b) são mostradas as curvas do tempo de processamento (em $\mu$ ) em função do número de SM por braço, no melhor e no pior caso, respectivamente. É possível observar que, tanto no melhor quanto no pior caso, os tempos de processamento de todos os métodos é bem próximo quando se tem 4 SM por braço. Entretanto, quando o número de SM aumenta, os desempenhos divergem. No melhor caso (Figura 10(a)), o método que apresentou melhor desempenho foi o método da inserção, enquanto o mais lento foi do método Quick. Os demais métodos apresentaram resultados bem próximos um do outro. Já no pior caso, Figura 10 (b), o melhor desempenho foi apresentado pelo método da seleção, enquanto os piores foram bolha, inserção e Quick, com aumento considerável no tempo de processamento. É importante mencionar que o tempo de processamento do método da seleção não mudou do melhor para o pior caso, ou seja, a disposição inicial dos elementos do vetor não interfere no desempenho desse método.

Figura 11 - Tempos de processamento do algoritmo de equalização com diferentes métodos de ordenação: (a) melhor caso e (b) pior caso

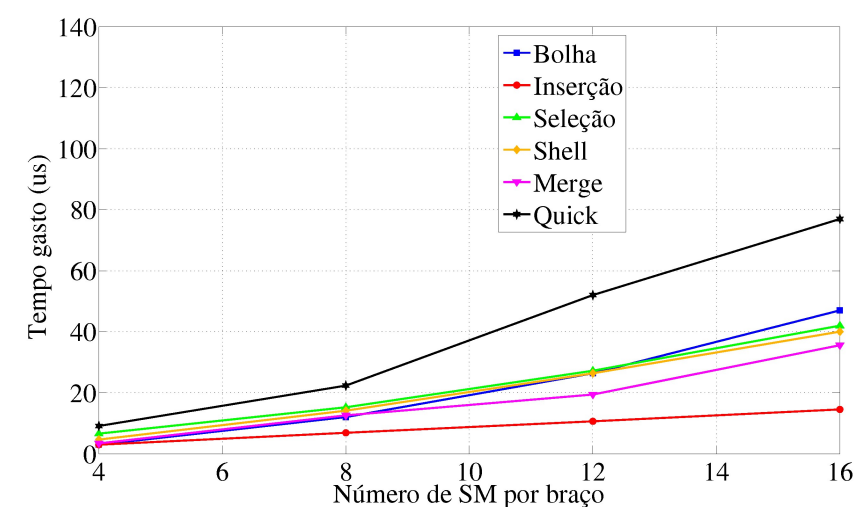

(a)

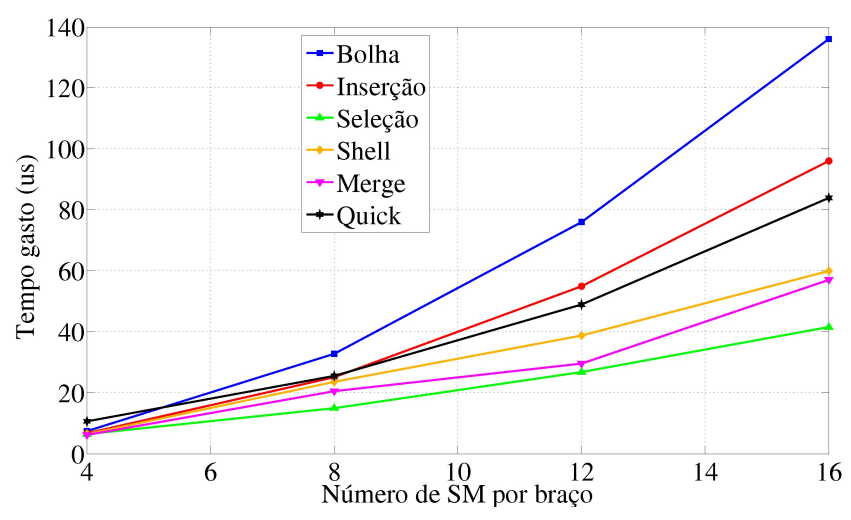

(b)

Fonte: Almeida et al (2016). 


\section{CONCLUSÕES FINAIS}

Neste artigo foram comparados os desempenhos de diferentes métodos de ordenação aplicados ao algoritmo de equalização das tensões dos capacitores do MMC. Para isso, foi verificado o funcionamento do algoritmo de equalização através de simulações, enquanto o esforço computacional de cada um dos métodos de ordenação aplicados a esse algoritmo foi verificado pela implementação em um DSP, onde o tempo de processamento pode ser medido. Foi possível verificar que para o vetor de tensões já ordenado, o método da inserção foi o mais rápido. Em contrapartida, com o vetor de tensões ordenado de forma inversa, o método da inserção foi o segundo pior, enquanto o método da seleção se mostrou mais rápido. Apesar de não ter sido o mais rápido no melhor caso, o método da seleção não alterou o tempo de processamento nos dois casos.

Os resultados obtidos com o DSP, mostrados na Figura 11, permitiram observar que quanto maior é o número de SM, maior é o tempo de processamento do algoritmo. Além disso, foi possível observar que o tempo de processamento de alguns algoritmos cresce de forma exponencial com o número de SM. Na prática, com o controle do conversor sendo feito em tempo real, o tempo de processamento pode influenciar diretamente no desempenho do sistema. Como o método da seleção apresentou crescimento linear e com a inclinação da curva menor que os demais, conclui-se que foi dele o melhor desempenho apresentado.

\section{AGRADECIMENTOS}

Este estudo foi financiado em parte pelo Conselho Nacional de Desenvolvimento Científico e Tecnológico (CNPq) - Proc. 456040/2014-5, Fundação de Amparo à Pesquisa do Estado de Minas Gerais (FAPEMIG) - Proc. APQ-01046-15, Coordenação de Aperfeiçoamento de Pessoal de Nível Superior - Brasil (CAPES) Código de Financiamento 001 e Universidade Federal de Juiz de Fora (UFJF).

\section{REFERÊNCIAS}

ALMEIDA, A. O.; GHETTI, F. T.; ALMEIDA, P. M.; BARBOSA, P. G. Desempenho de métodos de ordenação aplicados na equalização das tensões dos capacitores do conversor modular multinível. In: XXI Congresso Brasileiro de Automática, 2016, Vitória. Anais do XXI Congresso Brasileiro de Automática, 2016. v. 1. p. 570-576.

ARRILLAGA, J.; LIU, Y. H.; WATSON, N. R. Flexible power transmission: the HVDC options. Chichester, UK, John Wiley \& Sons, 2007.

BARNETT, G.; TONGO, L. D. Data Structures and Algorithms: Annotated Reference with 
Examples. Pierrefonds, CA, NETSlackers, 2008.

CARRASCO, J. M. et al. Power-electronic systems for the grid integration of renewable energy sources: A survey. IEEE Transactions on industrial electronics, v. 53, n. 4, p. 1002-1016, 2006.

DEBNATH, S. et al. Operation, control, and applications of the modular multilevel converter: A review. IEEE Transactions on Power Electronics, v. 30, n. 1, p. 37-53, 2015.

FEOFILOFF, P. Algoritmos em linguagem C. Rio de Janeiro, Brasil, Campus/Elsevier, 2009.

GLINKA, M. Prototype of multiphase modular-multilevel-converter with $2 \mathrm{MW}$ power rating and 17-level-output-voltage. In: IEEE 35th Annual Power Electronics Specialists Conference, Aachen, Germany. 2004. Proceedings of 35th Annual Power Electronics Specialists Conference, 2004. v. 4, p. 2572-2576.

INTERNATIONAL ELECTROTECHNICAL COMMISSION. IEC 61727: Photovoltaic (PV) Systems Characteristics of the Utility Interface. 2004.

INSTITUTE OF ELECTRICAL AND ELECTRONICS ENGINEERS. IEEE Std 929-2000

Recommended Practice for Utility Interface of Photovoltaic (PV) Systems. 2000.

LESNICAR, A.; MARQUARDT, R. An innovative modular multilevel converter topology suitable for a wide power range. In: IEEE Power Tech Conference, 2003, Bologna. Proceedings of Power Tech Conference, 2003. v. 3. p. 1-6.

MOHAN, N.; UNDELAND, T.; ROBBINS, W.P. Power Electronics: converters, applications and design. New Jersey, USA, John Wiley \& Sons, 2002.

RAJAN, M.; SEYEZHAI, R. Comparative study of multicarrier pwm techniques for a modular multilevel inverter. International Journal of Engineering and Technology (IJET), Citeseer, $v$. 5, n. 6, p. 4850-4865, 2013.

SAEEDIFARD, M.; IRAVANI, R. Dynamic performance of a modular multilevel Back-to-Back HVDC system. IEEE Transactions on Power Delivery, v. 25, n. 4,p. 2903-2912, 2010.

SHARIFABADI, K. et al. Design, control, and application of modular multilevel converters for HVDC transmission systems.Chichester, UK,John Wiley \& Sons, 2016.

TEODORESCU, R.; LISERRE, M.; RODRÍGUEZ, P. Grid Converters for Photovoltaic and Wind Power Systems. Chichester, UK, Wiley-IEEE Press, 2011.

YAZDANI, A.; IRAVANI, R. Voltage-sourced converters in power systems: modeling, control, and applications. New Jersey, USA, John Wiley \& Sons, 2010. 